\title{
On the proposal of a G0 phase and the restriction point
}

\author{
STEPHEN COOPER ${ }^{1}$ \\ Department of Microbiology and Immunology, University of Michigan Medical School, Ann Arbor \\ Michigan 49109-0620, USA
}

\begin{abstract}
Zetterberg and Larsson proposed that the restriction point divides the G1 phase into two parts. Cells before this point are able to leave the division cycle and enter a G0 phase; cells past this point are unaffected by a short period of low serum. Additional results of Zetterberg and Larsson-1) cycloheximide treatment affects cells in the same way as low serum, and 2) a delay in the second division after serum starvation in the cells not initially affected in their first division-indicate that their experiments are consistent with serum removal affecting cells in all phases of the cell cycle equally. Their experiments are consistent with the continuum model of the mammalian division cycle. There is no need to postulate a restriction point or a G0 phase to explain the serum starvation results.-Cooper, S. On the Proposal of a G0 phase and the restriction point. FASEB J. 12, 367-373 (1998)
\end{abstract}

Key Words: cell cycle $\cdot$ cell division $\cdot$ G1 phase

ZETTERBERG AND LARSSON (1) have summarized a large number of experiments proposing that the G1 phase of the mammalian cell division cycle could be subdivided into two phases. Cells from the early phase could enter the G0 phase, and those in the later phase could not (2). The point of division between these two phases was associated with the restriction point (3). I will present a reinterpretation of those experiments and provide an alternative explanation for the concept of the G0 phase and division of the G1 phase into different parts at the restriction point.

\section{ALTERNATIVE VIEWS OF THE CELL CYCLE}

There currently exist two diametrically opposed views of the regulation of the division cycle within the G1 phase. The dominant consensus or classical G1 regulatory model proposes that there are important regulatory functions, events, or stages in the G1 phase. For example, the G1 regulatory model proposes that cells choose, at some point in the G1 phase, whether to proceed through the remainder of the division cycle. Further, cells that differentiate decide to differentiate in the G1 phase of the division cycle. Most important, various G1-specific molecules are synthesized specifically within the G1 phase of the division cycle. Finally, genetic experiments indicate that there are genes specifically associated with passage through the G1 phase of the division cycle (4).

An alternative view of the division cycle questions the proposal of G1-specific events in mammalian cells. This alternative view proposes that the G1 phase exists when the time between the starts of the $S$ phases (i.e., the interinitiation time or the interdivision time) is greater than the sum of the $\mathrm{S}+\mathrm{G} 2+$ $\mathrm{M}$ phases. This view proposes that many experiments leading to the proposal of G1 events have different and equally valid interpretations. This alternative view proposes that one can account for the myriad results leading to the G1 event model by understanding the way many experiments have been overinterpreted. Previous publications have dealt with topics such as variability in the length of the G1 phase (5), the nature of the hypothesized 'G0 phase' (6), G1specific gene expression $(7,8)$, variation of the G1 phase during embryogenesis (9), analysis of complex results due to concanavalin A mitogenesis (10), and the G1 phase explanation of cycle variability (11). A complete analysis has been summarized in a book on the division cycle (12). This model has been christened the 'continuum' model to distinguish it from the 'classical' G1 control model of the mammalian division cycle.

This paper deals with a series of experiments that have been used to support the G1 model of the division cycle: the Zetterberg-Larsson experiments (1, $2)$. The appearance of this experiment in an important book devoted to the cell cycle (13) necessitates a clear and convincing reanalysis and reinterpretation. The experiments that will be analyzed here have been summarized (1), although the original data can be found in previously published articles $(2,14-16)$. A summary of the relationship of the proposals of Zetterberg and Larsson to other arrest points (restriction point, $\mathrm{W}$ point, etc.) is provided in a review article (17).

The Zetterberg-Larsson experiments are put forward as a precise experimental definition of the G0 phase of the cell cycle and strongly support the existence of a restriction point or decision point in the

\footnotetext{
${ }^{1}$ E-mail: cooper@umich.edu
} 


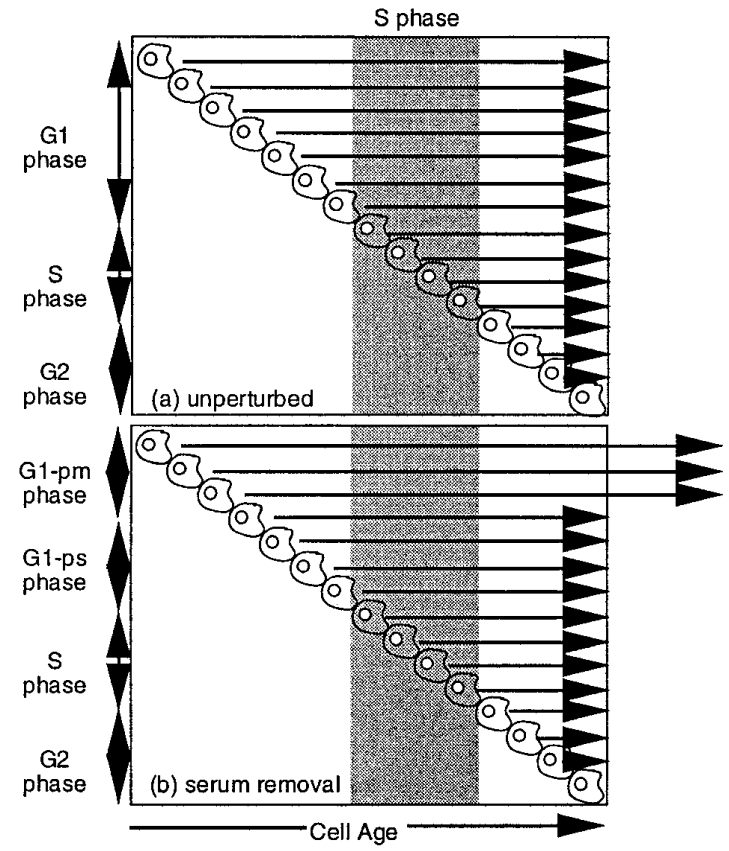

Figure 1. The basic Zetterberg-Larsson experiment. In panel $a$, cells of all ages are illustrated in an unperturbed, exponentially growing culture. Inasmuch as cell age is calculated from the time since it was produced by division, one can predict (disregarding statistical variation) the time of the next cell division. A cell will divide in a number of hours equal to the total interdivision time less the time since birth. At the start of the experiment, all cells are subjected to a short period (1 h) of serum starvation (time zero). The division time of each cell is determined. The results of serum starvation are illustrated in panel $b$. The youngest cells in culture (those in the proposed G1pm phase) have a delayed division, whereas all cells past a particular cell age divide on schedule. G1 phase cells delayed in division are proposed to be in a 'postmitotic' state $(\mathrm{Glpm})$; those later in the G1 phase (in these experiments, cells older than $3.5 \mathrm{~h}$ ) are in a 'presynthetic' (G1ps) phase of the division cycle.

mammalian G1 phase. Because the G0 phase is intimately related to control during the G1 phase of the cell cycle, the Zetterberg-Larsson experiments lend important support to the G1 control model of the cell cycle. As will be shown, these experiments are fully consistent with, and support, the continuum model.

\section{THE ZETTERBERG-LARSSON EXPERIMENT}

Cells growing exponentially in culture dishes were studied using a time-lapse video system. Zetterberg and Larsson observed cells growing and dividing over many generations. By noting when a particular cell arose by division, they knew the extant age of any cell in the culture at any particular time.

At a specific time, serum is removed from the cells; after $1 \mathrm{~h}$, the serum is restored. Time-lapse observation of the cells continues. The time until each cell will divide is determined, and changes from the nor- mal time of division are correlated with cell age at the time of serum removal. The basic observation is illustrated in Fig. 1. In a control condition (unperturbed culture with no serum removal; Fig. 1, upper panel), cells will divide so that all cell interdivision times are the same. Younger cells will take longer to divide than older cells. (In this analysis, normal cycle variability is not considered, and the results are presented in an idealized form.)

The effect of serum removal can be seen in the lower panel of Fig. 1. The youngest cells in the culture, those within $3.5 \mathrm{~h}$ of birth (said to be in the G1pm or 'postmitotic' phase), ${ }^{2}$ have a delayed division. Cells past this 3.5-h mark (G1 cells in this phase are called G1ps or presynthetic) have a normal, undelayed division. Cells in the S and G2 phases also have a normal, undelayed division. (Experimental determinations are summarized in Fig. 2a, $\boldsymbol{b}$.)

The division delay observed in the G1pm cells is approximately $8 \mathrm{~h}$, significantly longer than the 1-h serum removal. Zetterberg and Larsson proposed that cells early in the G1 phase (G1pm) are different from cells in the latter part of the G1 phase (G1ps). The G1pm cells can make a decision to leave the cell cycle and enter the G0 phase; G1ps cells cannot. The serum-starved G1pm cells enter the postulated G0 phase, and $8 \mathrm{~h}$ is required for the cells to return to the cell cycle. This proposal is illustrated in Fig. 3. The Zetterberg-Larsson results lead to the proposal that there is a particular point in the G1 phase at which cells change from G1pm to G1ps. Let us turn to these two separate ideas and analyze what is proposed.

G0

The G0 explanation of the extended 8-h delay after serum starvation is shown in Fig. 3. According to Zetterberg and Larsson, cells in a particular phase of the cycle, the G1pm cells, are able to enter G0. Cells past this phase are unable to enter G0. A detailed analysis of the G0 phase has been presented (12). The original postulation of an out-of-cycle G0 phase was made for liver cells in normal tissue. An extension of the G0 phase proposal to cells in tissue culture has been generally accepted without detailed proof that cultured cells truly model cells in normal tissue. The Zetterberg-Larsson experiment is thus a potentially precise definition of an out-of-cycle G0 phase. For this reason, one must critically analyze the results to see whether there is another explanation for the extended division delay observed in G1pm but not in G1ps cells.

\footnotetext{
${ }^{2}$ Abbreviations: G1pm, postmitotic phase; G1ps, presynthetic phase.
} 


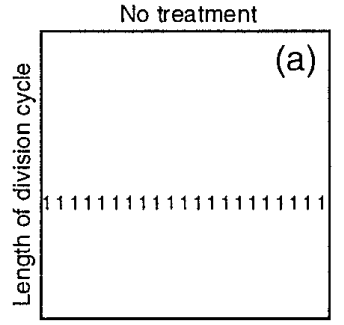

Cell age

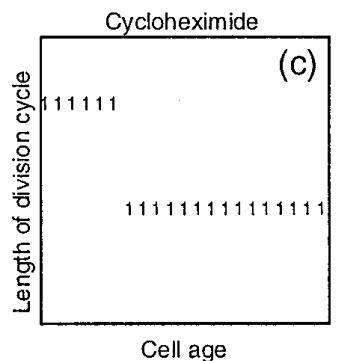

(C)

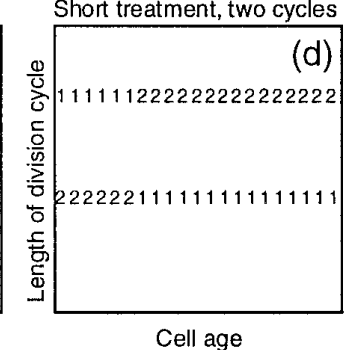

Figure 2. A formal display of the Zetterberg-Larsson results. The abscissa in each panel depicts the extant cell age at the time of treatment. The ordinate is the total interdivision time for cells of different extant ages. The numerals indicate whether the division plotted is the first or second interdivision time after treatment ( 1 for the first division cycle, 2 for the second division cycle). If there is no treatment ( $a)$, these control cells all have the same interdivision time; cells divide at the next division at the normal time. This is indicated by a 1 for the first division. A second division will occur by the superposition of a 2 over the other numerals, as all subsequent divisions will be of equal length. If there is a short period of serum withdrawal $(b)$, the cells of lower ages (left-most part of the abscissa) have an extended first division cycle. This is shown by the elevated numerals. Cells in the latter part of the division cycle have a normal division. Panel $a$ is a plotting of the results in Fig. $1 a$; panel $b$ plots the results of Fig. $1 b$. Panel $c$ ) indicates the results obtained after a short treatment with cycloheximide. Panel $d$ shows the first and second interdivision times resulting from a short period of serum withdrawal.

\section{THE RESTRICTION POINT}

The Zetterberg-Larsson proposal also implies that there is some decision point in the G1 phase of the division cycle when cells leave one particular statethose able to enter G0-and enter a different state. According to this explanation, a G1 event occurs. This event is then associated with a previously proposed point in the division cycle, the restriction point. Let us now turn to the restriction point and analyze how this applies to the Zetterberg-Larsson experiment.

Pardee $(3,18)$ proposed that the restriction point exists in mammalian cells. Cells starved of amino acids were observed to come to rest with a G1 amount of DNA. From experiments using different starvation protocols, Pardee proposed that there was a unique point within the G1 phase at which cells are arrested when growth is inhibited. Cells past this restriction point were able to initiate $S$ phase and ultimately divide. To be precise, there is no clear, convincing, and rigorous reason to associate the restriction point of Pardee with Zetterberg and Larsson's decision point between the G1pm and G1ps phases. That association is purely conjecture. But it is just this coalescence of ideas that makes the Zetterberg and Larsson experiment so convincing as a support of the G1 regulatory model of the cell cycle.

I will make this relationship more explicit. In Pardee's restriction point experiment, some cells in the G1 phase of the division cycle are able to initiate DNA synthesis even though they are still in G1 phase when an inhibitory condition is introduced (starvation for an amino acid, for example). Cells past a particular point can start $S$ phase and are not delayed. In the Zetterberg-Larsson experiment, there is a similar result, with cells past a particular G1 phase point able to initiate $S$ phase and ultimately divide. There is, on the surface, a strong similarity between the two experimental results.

\section{THE KEY QUESTION: DOES THE ZETTERBERG-LARSSON EXPERIMENT DEMONSTRATE THE EXISTENCE OF A G1-SPECIFIC DECISION POINT?}

The difference between the Zetterberg-Larsson analysis and the continuum model analysis of the Zetterberg-Larsson experiments rests on whether something specific happens during the G1 phase of the division cycle. There is no disagreement about the results, the observations, the methods, or even what happens when serum is removed from growing cells. The continuum model proposes that the results observed can be explained without invoking any G1-

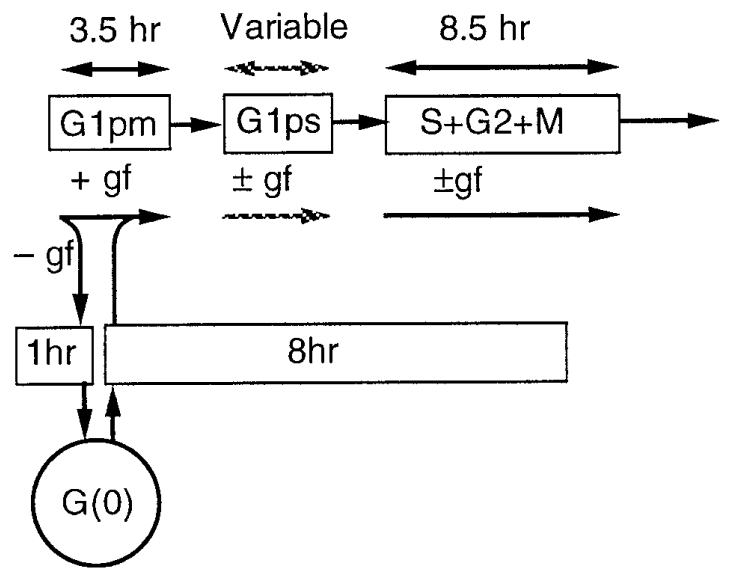

Figure 3. The G0 model of the Zetterberg-Larsson experiment. Cells in the G1pm phase are induced to leave the cycle and enter G0. Return from G0 takes $8 \mathrm{~h}$. (The '-gf' indicates removal of growth factors.) Cells past the decision point (or restriction point) between the G1pm and G1ps phases are unable to enter the G0 phase and thus do not have a delay. Removal of growth factors during the G1ps, S, G2, or M phases has no effect on division. 
specific controls, checkpoints, restriction points, or related phenomena. To see how this can be so, we have to look at two additional experiments performed by Zetterberg and Larsson, because these experiments indicate that the continuum model can explain the results as well as, if not better than, the $\mathrm{G} 0 /$ restriction point model.

The two experiments to be analyzed are the effect of cycloheximide on cell division and the observation of delay in the second division in the G1ps cells.

\section{CYCLOHEXIMIDE TREATMENT}

A summary of what happens when cells are untreated, starved of serum, or treated with cycloheximide is presented in Fig. 2. When cells are treated with cycloheximide, a delay in division of the youngest cells in the population is observed (Fig. 2c). This can be seen by comparing Fig. $2 c$ (cycloheximide treatment) with Fig. $2 b$ (serum removal). Cycloheximide is a chemical that inhibits protein synthesis in mammalian cells. I am aware of no evidence that cycloheximide acts in a cell cycle-specific manner. To the contrary, there is every reason to believe that cycloheximide affects protein synthesis in cells in all phases of the division cycle.

If we view the cycloheximide experiment not as an extension and elucidation of serum removal results, but as a control experiment, then a completely different interpretation arises. If the results of cycloheximide treatment were different from the serum removal experiment, then we could conclude that serum did something other than inhibit mass synthesis or protein synthesis in all phases of the division cycle. As a control experiment, however, one can conclude that cycloheximide inhibition does exactly the same thing as serum depletion. This means that serum withdrawal affects cells in all phases of the division cycle, but we get an observable effect only on the youngest cells of the division cycle.

How can we explain the difference between cells early and late in the G1 phase regarding either serum withdrawal or cycloheximide treatment? The continuum model explanation is presented in Fig. 4. This explanation is not meant to be definitive, but merely to show how an alternative explanation, without any cell cycle-specific effects, can give the appearance of a cell cycle-specific effect. The explanation proposed here is one counterexample of many that can be proposed. The continuum model postulates (in this hypothetical example) that removal of serum for a short period leads to an eventual delay of mass increase in cells. The effect of serum removal is not immediately seen, and mass increase continues for some period before reaching a plateau. There is a period before the effect of the 1-h starvation is felt by the cells. Cells of all ages experience the same pattern of mass in-

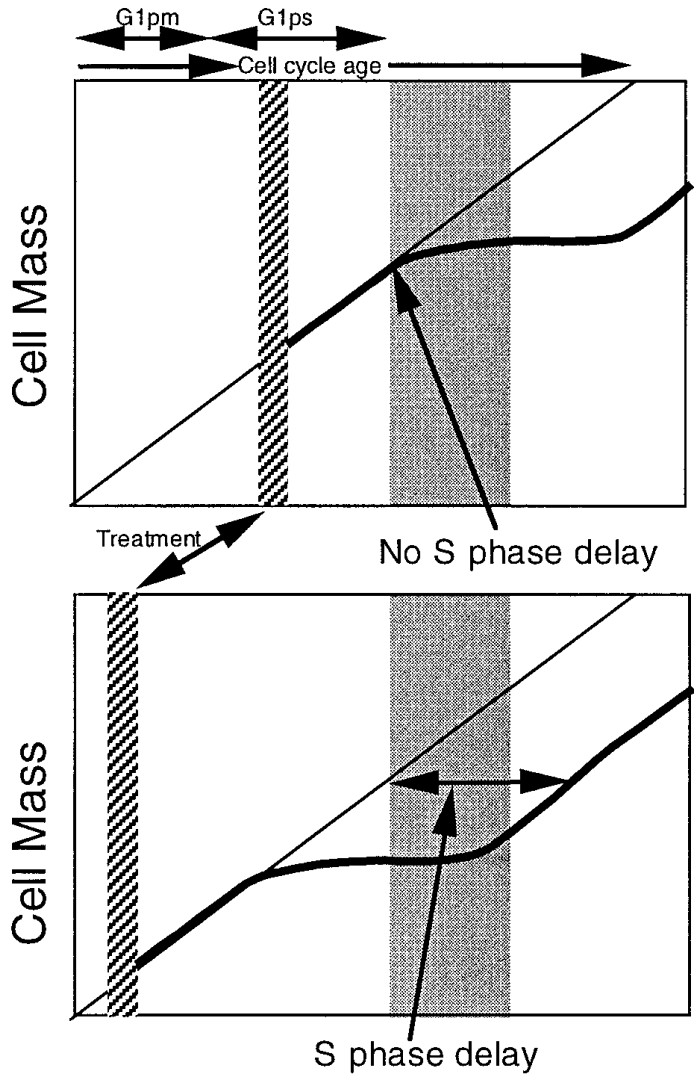

Figure 4. Leakage and entry into $S$ phase during serum starvation or cycloheximide treatment. The upper panel describes what happens to cells past the G1pm phase (i.e., cells in the Glps phase) when serum is removed for a short period of time (narrow striped area). Normally, mass would increase exponentially, as indicated by the straight-angled line. After a short period of serum starvation, it is possible that mass continues to increase in this exponential fashion. This soon stops. Then synthesis reaches a plateau that continues for an extended period of time. Cells that enter the $S$ phase (indicated by the shaded area) during this treatment are now defined as being in the Glps phase. These cells divide normally at the next division; no division delay is observed. In the lower panel, the same effect of serum withdrawal or cycloheximide treatment is observed on mass increase, but here we see that because the cells are further from achieving the mass related to initiation of $\mathrm{S}$ phase, they do not initiate $\mathrm{S}$ phase. Only at a later time, when mass increase resumes, will these cells achieve the normal mass associated with initiation of DNA synthesis, and S phase will begin.

crease when cells are treated with cycloheximide or when serum is withdrawn. The only difference is that cells can initiate $\mathrm{S}$ phase if they were close to starting it when a treatment (serum removal, cycloheximide addition) is applied. As shown in Fig. 4, cells close to the start of $\mathrm{S}$ phase can leak through and start $\mathrm{S}$ phase. Those cells requiring more mass increasecells earlier in the cell cycle and further from the start of $\mathrm{S}$ phase-are inhibited from starting a new $\mathrm{S}$ phase. Inhibited cells exhibit a delay in division due to a delay in the initiation of DNA synthesis.

The explanation given in Fig. 4 should not be taken as a precise 'prediction' of an experimental re- 


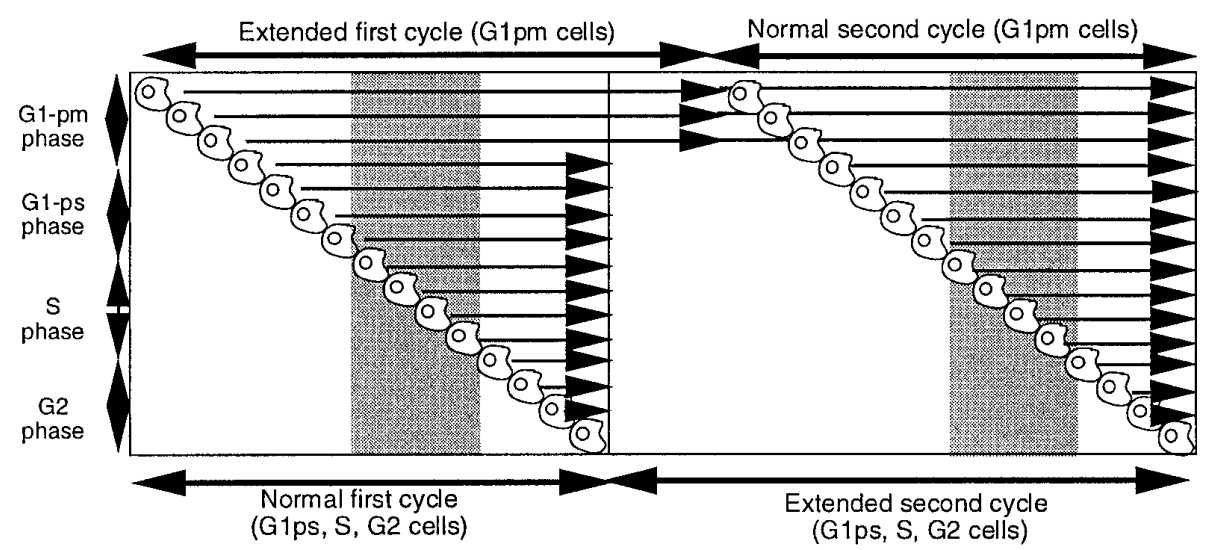

Figure 5. Analysis of two division cycles after short-term removal of serum. The results in Fig. 1 (lower panel) are repeated at the left, and a second cycle is examined at the right. After the first delayed division of the cells in the Glpm phase during serum removal, the second division occurs after a normal interval. In contrast, cells that were not delayed in the first cycle now have an extended second cycle. sult. Any general explanation where all cells in the culture are affected equally is acceptable. For example, the same result is obtained if all cells have a breakdown of some molecule and cells close to the start of S phase leak through; hence, a delay in the first division in the cells furthest from $\mathrm{S}$ phase. Figure 4 should be taken as a counterexample to indicate that one can explain the Zetterberg-Larsson experiments without invoking a cell cycle-specific mechanism.

Many years ago, a similar restriction point was proposed to exist in bacterial cells (19). Although it was not called a restriction point, it had all the defining properties of such a point. To make a long story short, an experimental analysis of the restriction point in bacteria (defined by chloramphenicol treatment) indicated that there was no restriction point $(20,21)$. The analysis presented here is strongly similar to the analysis of the bacterial restriction point presented more than 20 years ago. The only difference is that instead of bacteria and chloramphenicol, we have animal cells, serum withdrawal, and cycloheximide inhibition as the experimental elements. Yet the ideas are the same, and in both cases we can explain the phenomenon of the restriction point without resorting to any G1-specific phenomena.

\section{THE SECOND DIVISION DELAY}

After the original proposal of G1pm and G1ps states and the G0 phase phenomenon by Zetterberg and Larsson, subsequent experiments reported on the pattern of division after the initial division. Zetterberg and Larsson continued to follow cells until a second division occurred and determined the interdivision times of the second cycle. If cells in the G1ps, $\mathrm{S}$, and $\mathrm{G} 2 / \mathrm{M}$ phases were immune to a serum removal effect (indicated by the initial normal division), then we should expect no effect of serum on these cells even after the first division. If the continuum model is correct, then, in contrast, serum affects all cells in all phases of the division cycle. The con- tinuum model predicts that the second cycle would be delayed. Younger cells (in G1pm) that are affected in their first division cycle differ from the other cells, which are either in the $S$ and G2/M phases of the division cycle or close enough to the start of $S$ phase (in the G1ps phase) to be able to leak through to start S phase. Once S phase starts, the cell can divide. Whatever effect serum has on the growth of the cell will be exhibited in the second cycle.

This is evident in the results of Larsson, Zetterberg, and Engstrom (16), as shown in Fig. $2 d$. The second division is delayed in the same manner as the first division of the presumed G1pm cells. After two division cycles, all cells are back on track and no further delays are expected to be observed. A more extensive illustration of the delay phenomenon is presented in Fig. 5, where the results of Fig. 1 are extended to a second cycle. Figure 6 analyzes the experiment in terms of the pattern of mass synthesis in all cells in the culture that initially escape division delay and undergo a second delay.

\section{THE CONTINUUM MODEL EXPLANATION OF THE ZETTERBERG-LARSSON EXPERIMENT}

Exponentially growing cells synthesize mass throughout the division cycle. This mass may be made up of specific trigger molecules related to the initiation of $\mathrm{S}$ phase. These molecules, like total mass, are made continuously throughout the division cycle. It is proposed that such trigger molecules are a constant fraction of cell mass. Stated another way, any specific initiator of $S$ phase can be considered a constant fraction of cell mass, throughout the division cycle and without any cycle-specific pattern of synthesis, to give the observed results of the Zetterberg-Larsson experiments.

The continuum explanation of such 'leakage' is illustrated in Fig. 6. If there were no intervention (e.g., serum removal), then mass synthesis would continue along the exponential line. The removal of serum, 


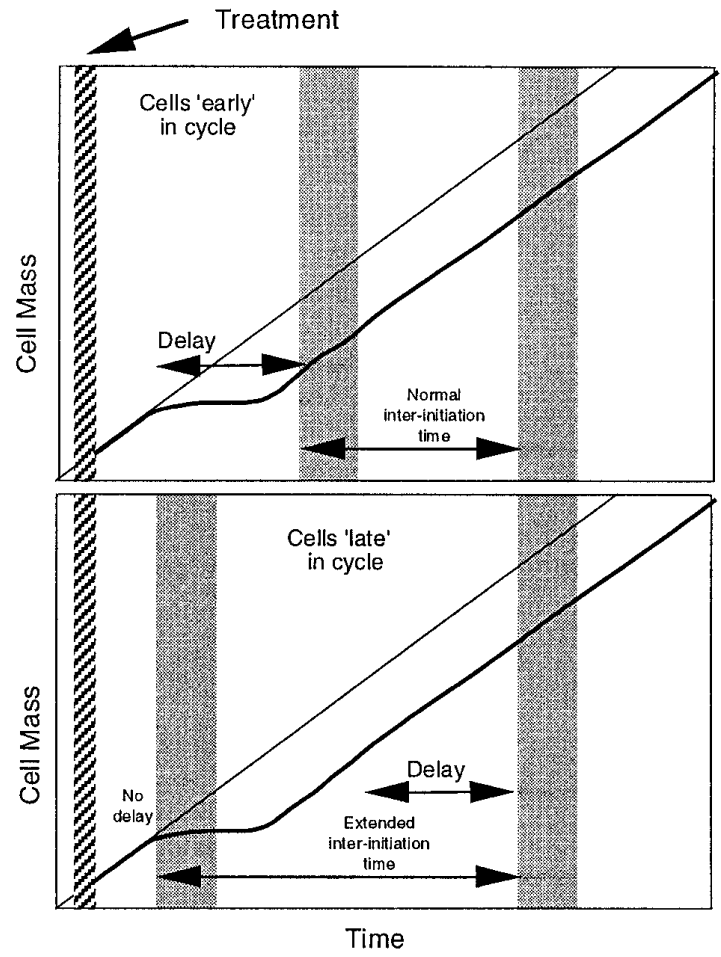

Figure 6. Explanation of second division delay in treated G1ps cells; analysis of Fig. 4 is extended to a second division. The pattern of deviation of mass increase is identical in both panels. Cells in any phase of the division cycle have the same pattern of continued synthesis: a plateau giving a delay in mass increase and recovery to the original rate of mass synthesis. Cells that escape the initial inhibition (cells past the Glpm phase) have a second division delay.

the continuum model proposes, has a long-term effect on mass synthesis, although this effect occurs after a short period of continuing mass synthesis. The long plateau would be expected to delay the increase in mass synthesis by $\approx 8 \mathrm{~h}$. Cells early in the cycle delay the first initiation of $S$ phase, and cells that initiate DNA synthesis normally (by leakage through to initiation mass) will delay the second cycle. No complex explanations using a proposed G0, proposed restriction points, or proposed G1-specific events are required.

\section{MOLECULAR BASIS OF INITIATION}

The use of mass as an indicator of initiation potential is merely a shorthand approach to the problem. If one wished to substitute "a specific initiator molecule that was made continuously during the division cycle and was present as a constant proportion of cell mass," there would be no fundamental problem. The notion of initiation mass, so well worked out in bacteria, is merely a convenient concept with which to discuss the nature of the division cycle. What must be recognized is that if mass is made continuously during the division cycle and not restricted to a partic- ular phase of the division cycle, then the same can be said for any initiator molecule.

\section{PREDICTIONS OF THE CONTINUUM MODEL}

The hallmark of a scientific model is that it makes predictions that can be verified by experiment. Here a prediction will be made that specifically applies to the Zetterberg-Larsson experiment. The question is whether the particular point defined as being $3.5 \mathrm{~h}$ after division in the G1 phase is really a biologically significant cell cycle marker or merely an experimental artifact. The continuum model predicts that if the cycloheximide concentration were varied around the concentration used in the Zetterberg-Larsson experiments and continuously increased and decreased, the particular point of demarcation would vary. As cycloheximide concentration increased, the G1pm phase would increase (i.e., more cells would have a delayed first division) and the G1ps phase would thus decrease. As cycloheximide concentration decreased, the G1pm phase would decrease (i.e., fewer cells would have a delayed first division) and the G1ps phase would increase. This is because the postulated leakage would increase with decreasing cycloheximide and decrease with increasing cycloheximide. If varying cycloheximide concentrations yielded the predicted variation in the time when cells left the G1pm phase, then to salvage the G0 model one would have to postulate that there exist an infinite number of G0 states. This is reminiscent of the earlier postulation of deeper and deeper states of G0 produced by different times of serum starvation (22). Furthermore, these experiments are reminiscent of much earlier bacterial experiments in which chloramphenicol was varied over a wide range such that one had to choose between a leakage model and a restriction point model with an infinite number of restriction points. In the bacterial case, it was possible to demonstrate experimentally that the leakage explanation was clearly the correct one $(20,21)$.

The same prediction can be made for the serum treatment, with different periods of starvation or starvation serum levels (i.e., different amounts of residual serum during the starvation period), leading to different delay periods for G1pm cells, or with the G1pm period being shorter or longer.

\section{THE NATURE OF THE DIVISION GYCLE}

The continuum model does not propose the existence of G1-specific events, checkpoints, restriction points, or decision points. No unique G1-specific decision points occur that are specific to G1 phase. Things may happen elsewhere ( $\mathrm{S}$ or G2/M phases) that do not happen in G1 phase, but not the reverse. 
The G1 phase exists merely because the time for $\mathrm{S}$ and $\mathrm{G} 2 / \mathrm{M}$ phase is less than the time between the starts of S phases. There are G1-less cells (23), and such cells are completely consistent with the continuum model. It should be understood that the continuum model was developed to explain the absence of G1 phase in some animal cells and the existence of a G1 phase in bacterial cells (5).

Here we have taken a crucial set of experiments that have been used to support the G1 model of the cell cycle and have shown by analysis that there is no need to postulate a G1-specific decision point. The treatments studied by Zetterberg and Larsson affect all cells equally. For reasons that are easily understood, we can say that the Zetterberg and Larsson experiments are fully consistent with the postulates of the continuum model. No support of the restriction point or the G0 phase is found in the experiments of Zetterberg and Larsson. The ideas presented here are not completely new. They have been said before, but have been forgotten. One early example from a paper published in 1975 (24) put it very well:

"Up to $96 \%$ of the cells in postconfluent cultures growing in conventional medium become labeled upon continuous, prolonged exposure to ${ }^{3} \mathrm{H}$-thymidine. Seventy-eight percent of the cells in serum-deprived cultures growing at a very low rate become labeled. These and other considerations suggest that the inhibition of cell multiplication by high population density or serum deprivation is caused by a lengthening of the time cells remain in the pre-replicative G1 period rather than by shifting cells into a qualitatively distinct G0 period."

I want to particularly thank Charles Helmstetter and Jay Keasling, who were both extremely helpful with suggestions and comments. Alexandra Cooper, as ever, is a devoted editor who made this paper clearer, shorter, and much better than it would have been otherwise. This paper is dedicated to Dr. Harry Rubin, who was so clear and understanding in his critique of the G0 proposal.

\section{REFERENCES}

1. Zetterberg, A., and Larsson, O. (1995) Cell cycle progression and cell growth in mammalian cells: kinetic aspects of transition events. In Cell Cycle Control (Hutchinson, C., and Glover, D. M., eds) pp. 206-227, Oxford University Press, Oxford, New York
2. Zetterberg, A., and Larsson, O. (1985) Kinetic analysis of regulatory events in G1 leading to proliferation or quiescence of Swiss 3T3 cells. Proc. Natl. Acad. Sci. USA 82, 5365-5369

3. Pardee, A. B. (1974) A restriction point for control of normal animal cell proliferation. Proc. Natl. Acad. Sci. USA 71, 1286-1290

4. Murray, A., and Hunt, T. (1993) The Cell Cycle, W. H. Freeman and Company, New York

5. Cooper, S. (1979) A unifying model for the G1 period in prokaryotes and eukaryotes. Nature (London) 280, 17-19

6. Cooper, S. (1981) The continuum model: application to G1-arrest and G0, In Cell Growth (Nicolini, C., ed) pp. 315-336, Plenum Press, New York

7. Cooper, S. (1989) The continumum model and c-myc regulation. J. Theor. Biol. 135, 393-400

8. Okuda, A., and Cooper, S. (1989) The continuum model: an experimental and theoretical challenge to the G1-model of cell cycle regulation. Exp. Cell Res. 185, 1-7

9. Cooper, S. (1981) The central dogma of cell biology. Cell Biol. Int. Rep. 5, 539-551

10. Guiguet, M., and Cooper, S. (1982) A critique of the use of DNA synthesis as a measure of the effect of mitogens on lymphocytes. Biosci. Rep. 2, 91-98

11. Cooper, S. (1982) The continuum model: statistical implications. J. Theor. Biol. 94, 783-803

12. Cooper, S. (1991) Bacterial Growth and Division, Biochemistry and Regulation of Prokaryotic and Eukaryotic Division Cycles, Academic Press, San Diego, California

13. Hutchinson, C., and Glover, D. M. (1995) Cell Cycle Control, Oxford University Press, Oxford, New York

14. Zetterberg, A., and Larsson, O. (1991) Coordination between cell growth and cell cycle transit in animal cells. Cold Spring Harbor Symp. Quant. Biol. 56, 137-147

15. Larsson, O., Dafgard, E., Engstrom, W., and Zetterberg, A. (1987) Immediate effects of serum depletion on dissociation between growth in size and cell division in proliferating 3T3-cells. J. Cell Physiol. 127, 267-273

16. Larsson, O., Zetterberg, A., and Engstrom, W. (1985) Consequences of parental exposure to serum-free medium for progeny cell division. J. Cell Sci. 75, 259-268

17. Norbury, C., and Nurse, P. (1992) Animal cell cycles and their control. Annu. Rev. Biochem. 61, 441-470

18. Pardee, A. B. (1989) G1 events and regulation of cell proliferation. Science 246, 603-608

19. Lark, K. G., and Renger, H. (1969) Initiation of DNA replication in Escherichia coli 15T: Chronological dissection of three physiological processes required for initiation. J. Mol. Biol. 42, 221-235

20. Cooper, S. (1974) On a criterion for using chloramphenicol to define different processes in the initiation of DNA synthesis in bacteria. J. Theor. Biol. 46, 117-127

21. Cooper, S., and Wuesthoff, G. (1971) Comment on the use of chloramphenicol to study the initiation of deoxyribonucleic acid synthesis. J. Bacteriol. 106, 709-711

22. Augenlicht, L., and Baserga, R. (1974) Changes in the G0 state of WI-38 fibroblasts at different times after confluence. Exp. Cell. Res. 89, 255-262

23. Liskay, R. M., and Prescott, D. M. (1978) Genetic analysis of the G1 period: Isolation of mutants (or variants) with a G1 period from a Chinese hamster cell line lacking G1. Proc. Natl. Acad. Sci. USA 75, 2873-2877

24. Rubin, H., and Steiner, R. (1975) Reversible alterations in chick embryo mitotic cycle in various states of growth regulation. J. Cell Physiol. 85, 261-270

Received for publication October 20, 1997. Accepted for publication November 5, 1997. 